Article

\title{
Application of an In Vitro Psoriatic Skin Model to Study Cutaneous Metabolization of Tazarotene
}

\author{
Alexandre Morin ${ }^{1,2,+}$, Mélissa Simard ${ }^{1,2,+}$, Geneviève Rioux ${ }^{1,2}$, Alexe Grenier ${ }^{1,2}$, \\ Sophie Morin ${ }^{1,2}$ and Roxane Pouliot $1,2, *$ (D) \\ 1 Centre de Recherche en Organogénèse Expérimentale de l'Université Laval/LOEX, Axe Médecine \\ Régénératrice, Centre de Recherche du CHU de Québec-Université Laval, Québec, QC G1J 1Z4, Canada; \\ ax_mo@hotmail.com (A.M.); melissa.simard.6@ulaval.ca (M.S.); genevieve.rioux.9@ulaval.ca (G.R.); \\ alexe.grenier.1@ulaval.ca (A.G.); sophie.morin.7@ulaval.ca (S.M.) \\ 2 Faculté de Pharmacie, Université Laval, Québec, QC G1V 0A6, Canada \\ * Correspondence: roxane.pouliot@pha.ulaval.ca; Tel.: +1-418-525-4444 (ext. 61706) \\ + These two authors contributed equally to the work and therefore should be considered as equivalent \\ first authors.
}

Received: 10 October 2019; Accepted: 18 November 2019; Published: 21 November 2019

\begin{abstract}
Psoriasis is an inflammatory skin disease characterized by the presence of whitish and scaly plaques, which can cover up to $90 \%$ of the body surface. These plaques result from the hyperproliferation and abnormal differentiation of keratinocytes. Dermopharmaceutical testing of new therapies is limited by healthy and pathological skin models, which are not closely enough mimicking their in vivo counterparts. In this study, we exploited percutaneous absorption and Ultra Performance Liquid Chromatography (UPLC) analyses in order to determine the metabolic capacity of our psoriatic skin model. Skin substitutes were reconstructed according to the self-assembly method and tested regarding their percutaneous absorption of a topical formulation of tazarotene, followed by UPLC analyses. Histological and immunofluorescence analyses confirmed both the healthy and psoriatic phenotypes. Results from percutaneous absorption showed a significant level of tazarotene metabolite (tazarotenic acid) when the formulation was applied over $24 \mathrm{~h}$ on the skin substitutes. The presence of tazarotenic acid in the dermis and the epidermis of healthy and psoriatic skin substitutes confirms the metabolic capacity of both skin models, and thereby their ability to screen new molecules with antipsoriatic potential. In conclusion, the present data suggest that our psoriatic skin model could possibly be used in clinic to screen in vitro responses of patient to a panel of drugs without having them experiencing the drawback of each drug.
\end{abstract}

Keywords: tissue engineering; 3D culture; tazarotene; psoriasis; metabolization; skin substitutes

\section{Introduction}

Psoriasis is a chronic inflammatory disease which affects 2 to $3 \%$ of the world population, both men and women [1]. It is characterized by the presence of whitish and scaly plaques that can be found on every body part [2]. Those plaques are the result of the abnormal differentiation and hyperproliferation of keratinocytes, which leads to a thicker epidermis (acanthosis) [3-5]. Psoriasis is also characterized by the infiltration of immune cells, increased angiogenesis, and the retention of nuclei by corneocytes (parakeratosis) [6,7]. In this pathology many genes have their expression modified, including differentiation, proliferation, and inflammation markers. Myeloid-related protein 8 (MRP8), involucrin, filaggrin, keratins, and transcription factor activator protein 1 (AP-1) are all examples of proteins whose gene expression is altered [8]. It is known that psoriasis is a multifactorial disease including genetic susceptibility and environmental factors, but the cause of psoriasis still remains unknown $[9,10]$. 
Since the etiology of psoriasis remains largely unknown, and patients develop various manifestations of the disease, from light and small to deep and large plaques with different characteristics, the treatment of this disease remains highly empirical. Indeed, psoriasis has no known curative treatment; therapy aims to reduce symptoms and improve quality of life. The choice of treatment for psoriasis depends on many factors, including the extent of the disease, its effect on a patient's life, and the patient's perception of his illness [6]. In a population-based survey of 3426 patients and 781 physicians in North America and Europe, $46 \%$ of patients agreed that using currently available therapies for psoriasis and psoriatic arthritis can be worse than the condition itself, and $85 \%$ felt that there is a need for better therapies [11]. Difficulties in prescribing the best treatment for psoriasis patients are a major concern since a lot of inter-individual variability is observed and therefore patients respond very differently to the large panel of available drugs [12].

Among the molecules currently used to treat psoriasis, retinoids present good effectiveness mainly if they are used in combination with corticosteroids [13]. Tazarotene is an acetylenic retinoid with a low systemic bioavailability, which is used in cream or gel forms as a topical treatment [14]. Tazarotene is hydrolyzed into tazarotenic acid by an esterase produced by keratinocytes (Figure 1) $[15,16]$. Once tazarotenic acid is metabolized, it specifically binds retinoic acid receptors (RARs) RAR- $\beta$ and RAR- $\gamma$ and then forms homodimers and heterodimers (RAR-RAR). Once the complex is formed, it can regulate the expression of tumor suppressors, tazarotene-induced genes, or form a complex with AP-1 to normalize the expression of differentiation and proliferation markers [17-19]. Even if it has a lower toxicity than most other retinoids, some adverse effects can be observed, such as irritation and burns [16].
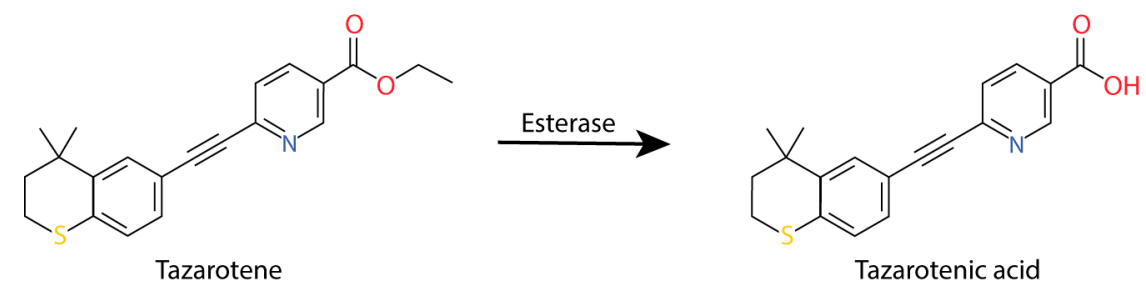

Figure 1. Tazarotene metabolization into tazarotenic acid.

Nowadays, restrictions on and security concerns about the use of humans and animals in cosmetic and pharmaceutical research necessitate the development of other models such as in vitro skin substitutes. The substitutes reconstructed in our laboratory according to the self-assembly method are free of exogenous materials. Moreover, they can be easily adapted for the reconstruction of skin substitutes having many of the features of a psoriatic skin phenotype. However, there is almost no information on the skin substitute metabolism. More detailed knowledge of this metabolism could help researchers to ensure that psoriatic skin substitutes represent a good model for molecule screening and open the door to more personalized medicine.

In this study, the objective was to characterize the psoriatic skin substitutes' metabolism of tazarotene. Three types of samples, normal human skin (NHS), healthy substitutes (HS), and psoriatic substitutes (PS), were tested to compare their permeability and structures. The percutaneous absorption method was used to determine the metabolic capacity and UPLC was used to quantify the amount of tazarotene and its metabolite (tazarotenic acid) found in each layer of the samples.

\section{Materials and Methods}

\subsection{Patients}

This study was conducted in agreement with the Helsinki Declaration and performed under the guidelines of the research ethics committee of the "Centre hospitalier universitaire de Québec" (ethic code: DR-002-1121 protocol renewal approved on 30 January 2019). All patients were given adequate information to provide written consent. The subjects were Caucasian males and females aged between 
22 and 64 years old from our cell bank (cell populations: 22, 38, and 46 years old (healthy) and 47, 49, and 64 years old (psoriatic)). Biopsies used as controls were obtained from breast lift surgeries. Subjects were Caucasian females aged between 31 and 41 (healthy skin biopsies: 31, 38, 39, and 41 years old).

\subsection{Cell Culture Media}

Fibroblasts were cultured in the Dulbecco-Vogt modification of Eagle's medium (DMEM) supplemented with 10\% fetal calf serum (Invitrogen, Burlington, ON, Canada), $100 \mathrm{UI} / \mathrm{ml}$ penicillin (Sigma Chemicals, St-Louis, MO, USA), and $25 \mu \mathrm{g} / \mathrm{mL}$ gentamicin (Schering, Pointe-Claire, QC, Canada). Keratinocytes were cultured in a combination of DMEM with Ham's F12 (3:1) supplemented with $5 \%$ Fetal Clone II serum (Hyclone, Scarborough, ON, Canada), $5 \mu \mathrm{g} / \mathrm{mL}$ insulin (Sigma), $0.4 \mu \mathrm{g} / \mathrm{mL}$ hydrocortisone (Cedarlane, Hornby, ON, Canada), $10^{-10} \mathrm{M}$ cholera toxin (ICN Biochemical, Montréal, QC, Canada), $10 \mathrm{ng} / \mathrm{mL}$ human epidermal growth factor (EGF) (Austral Biological, San Ramon, CA, USA), $100 \mathrm{UI} / \mathrm{mL}$ penicillin (Sigma), and $25 \mu \mathrm{g} / \mathrm{mL}$ gentamicin (Schering).

\subsection{Cell Culture}

Keratinocytes were extracted from skin biopsies using the isolation method with thermolysin and trypsin [20]. Keratinocytes (passage one) were seeded at $8 \times 10^{3} \mathrm{cells} / \mathrm{cm}^{2}$ on a feeder layer of irradiated 3T3 mouse fibroblasts. Fibroblasts were extracted from skin biopsies using the isolation method with thermolysin and collagenase [21]. They were seeded at $4 \times 10^{3} \mathrm{cells} / \mathrm{cm}^{2}$ and used at passage six for skin substitute production. All cultures were incubated at $37^{\circ} \mathrm{C}$ in an $8 \% \mathrm{CO}_{2}$ air atmosphere and changed three times a week with the media previously described in the cell culture media section. Cells were frozen in liquid nitrogen until needed.

\subsection{Production of Tissue-Engineered Substitutes}

Skin substitutes were reconstructed according to the self-assembly method. Briefly, fibroblasts were cultured in the presence of ascorbic acid at a concentration of $50 \mu \mathrm{g} / \mathrm{mL}$ (Sigma) to form manipulatable sheets. After 28 days, dermal sheets were carefully detached from the flasks with curved forceps and superimposed. After a week, the keratinocytes (passage two) were seeded on the dermal equivalent to form a new epidermal layer. After seven days of cell proliferation, the substitutes were raised to the air-liquid interface and cultured for 21 days. All culture media were changed three times a week.

\subsection{Histological Analyses}

Three skin substitute biopsies of each cell population were fixed in HistoChoice ${ }^{\circledR}$ solution and embedded in paraffin wax. Five-micrometer-thick sections were cut and stained with Masson's trichrome. From the histological analyses, the living epidermal thickness was measured using ImageJ software. Five measurements in three different sections of each biopsy were made.

\subsection{Immunofluorescence Analyses}

After 21 days of culture at the air-liquid interface, biopsies were taken and the samples were embedded in Tissue-Tek O.C.T. compound (Somagen Diagnostics Inc., Edmonton, AB, Canada) to be stored at $-80^{\circ} \mathrm{C}$ until needed. Indirect immunofluorescence analyses were performed on acetone-fixed cryosections (5- $\mu \mathrm{m}$ thick). Frozen sections of normal human skin were used as positive controls. The following primary antibodies were used: rabbit anti-keratin 14 (IgG1) (dilution 1:1600, Cedarlane), mouse anti-involucrin (IgG1) (dilution 1:800; Sigma), and mouse monoclonal (IgG1) anti-human filaggrin (dilution 1:800; BTI, Stoughton, MA, USA). Then, tissues were incubated with Alexa 594 goat anti-mouse IgG $(\mathrm{H}+\mathrm{L})$ (dilution 1:400; Molecular Probes, Eugene, OR, USA) or Alexa 488 donkey anti-rabbit IgG $(\mathrm{H}+\mathrm{L})$ (dilution 1:1000, Molecular Probes). The nuclei were labeled with Hoechst reagent 33258 (dilution 1:100; Sigma) and added to the secondary antibody solution. Twenty fields of each combination were counted (magnification 40). 


\subsection{Percutaneous Absorption}

Percutaneous absorption was measured using the standard Franz diffusion cell apparatus as described by Franz [22,23]. Briefly, samples (NHS, HS, or PS) were clamped tightly between the two glass chambers of the diffusion cell $\left(0.636 \mathrm{~cm}^{2}\right)$. The receptor compartment was filled with medium (Phosphate Buffer Saline [PBS] and ethanol [80/20; v/v]) and maintained at $37^{\circ} \mathrm{C}$ with a heat block. Ten $\mathrm{mg}$ of Tazorac ${ }^{\circledR}$ cream $0.1 \%$ (Allergen Inc., Markham, ON, Canada), corresponding to $10 \mu \mathrm{g}$ of tazarotene, were deposited on each skin. Receptor medium samples were taken at intervals $(2,4,8$, and $24 \mathrm{~h}$ ) with a $5 \mathrm{~mL}$ syringe lengthened by a catheter, and were conserved at room temperature until needed. When the kinetic assay was finished, the skin surface was washed with a solution of methanol $(100 \%)$, which was collected for analysis. Then, the epidermis and the dermis were separated and were put in a solution of methanol $(100 \%)$. The samples were analyzed by ultra performance liquid chromatography (Acquity UPLC H-Class System, Waters, Mississauga, ON, Canada) with a BEH C18 column (Waters, Mississauga, ON, Canada) using the fluorescence detector. The mobile phase was methanol $(100 \%)$ and $\mathrm{NaH}_{2} \mathrm{PO}_{4}$ at $\mathrm{pH} 2.5$ with $\mathrm{H}_{4} \mathrm{PO}_{4}$ at a flow rate of $0.5 \mathrm{~mL} / \mathrm{min}$; the molecules were detected at $359 \mathrm{~nm}$ and $505 \mathrm{~nm}$. A calibration curve was used to quantify the amount of tazarotene and tazarotenic acid that diffused through the skin.

\subsection{Statistical Analyses}

Data were expressed as mean \pm standard deviation (SD). Statistical analyses of the living epidermis thickness were performed using an unpaired Student t-test. Percutaneous absorption results were analyzed using two-way ANOVAs followed by Tukey's post-hoc tests. Results were considered significant when the p-value was less than 0.05 . Statistical analyses were performed with GraphPad Prism software version 8.1 (GraphPad Software Inc. San Diego, CA, USA).

\section{Results}

\subsection{Morphology of the Psoriatic Skin Substitutes Produced by Tissue Engineering}

Macroscopic analyses showed that HS (Figure 2a) had a regular aspect on the majority of the sample surface, with a smooth and uniform aspect, suggesting a normal epidermal differentiation and a normal distribution all over the surface. At the opposite, their psoriatic counterparts (Figure 2b) showed a more irregular and scaly aspect, suggesting an abnormal epidermal differentiation and proliferation. The distribution was very scattered as seen with psoriatic plaques in vivo.

Masson's trichrome staining was performed to validate the skin phenotype. HS showed all the normal skin layers with a well-defined stratum corneum (Figure 2c). Psoriatic substitutes showed a thicker and less organized epidermis than the control (Figure 2d). The stratum corneum was disorganized or even absent on most of them. Measures of the living epidermis thickness (Figure 2e) confirmed that PS have a thicker epidermis than HS.

The pathological phenotype of psoriatic substitutes (Figure 3g-i), in contrast to NHS (Figure 3a-c) and HS (Figure $3 \mathrm{~d}-\mathrm{f}$ ), was confirmed by immunofluorescence staining of proteins altered in the psoriasis differentiation process. Involucrin expression was mainly localized in the upper layers of NHS and HS while it was found throughout the epidermis of PS (Figure 3a,d,g). In HS, filaggrin was expressed in the granular layer with greater intensity compared to the PS (Figure 3e,h). The same intensity was observed between NHS and HS (Figure 3b,e). The expression of keratin 14 appeared predominantly in the basal layer of both NHS and HS and was overexpressed in the PS (Figure $3 c, f, i)$. All these results are in accordance with in vivo expression, showing a more disturbed epidermal differentiation in PS than in HS, which is characteristic of the pathology [24-26]. 

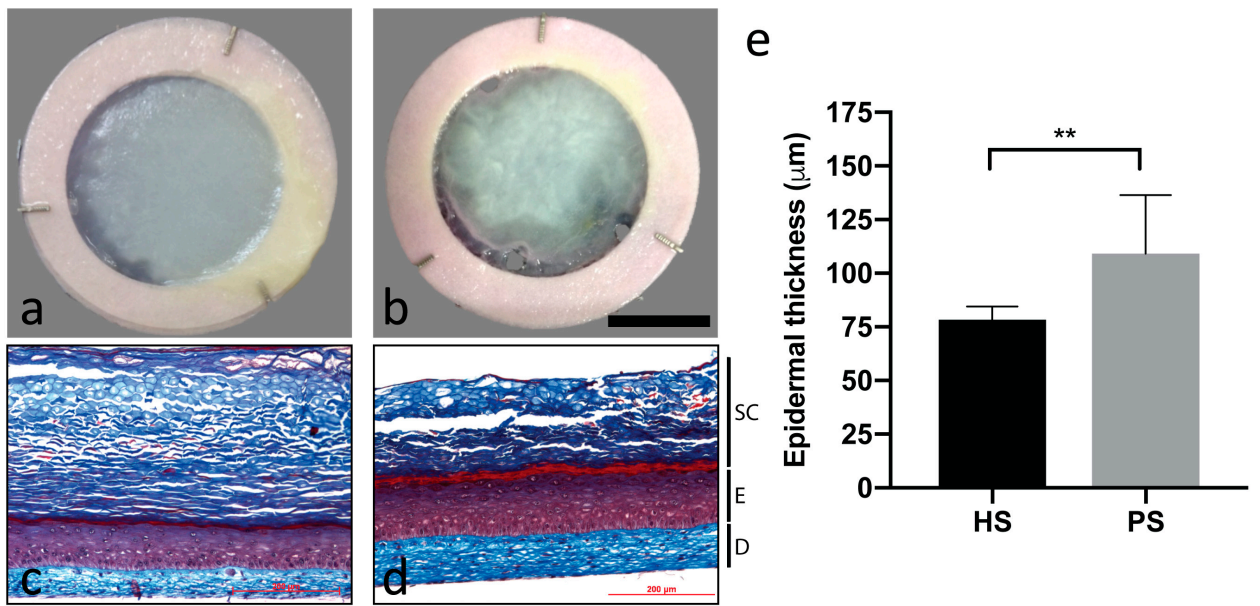

Figure 2. Macroscopic aspect of healthy substitutes (HS) (a) and psoriatic substitutes (PS) (b) as well as histological analyses of Masson's trichrome staining of HS (c) and PS (d) and the living epidermis thickness measurements (e) of these skin substitutes. Scale bar: $a, b=1 \mathrm{~cm} ; \mathrm{c}, \mathrm{d}=200 \mu \mathrm{m}$. Fibroblasts and keratinocytes from four different healthy donors and four different psoriatic donors were used ( $\mathrm{n}=$ three substitutes per donor). SC: Stratum corneum; E: Epidermis; and D: Dermis. Unpaired Student t-test, ${ }^{*} p<0.05 ;{ }^{* *} p<0.01$.

Involucrin

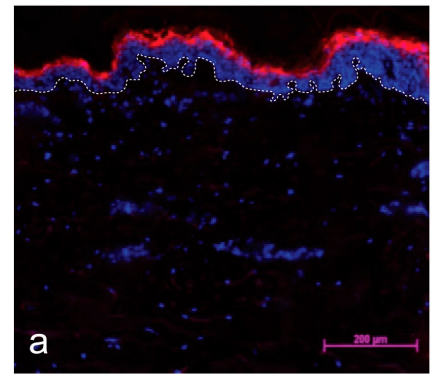

HS
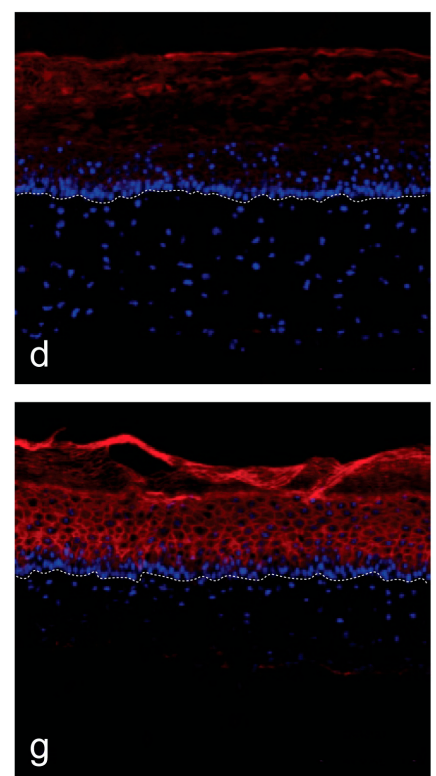

Filaggrin
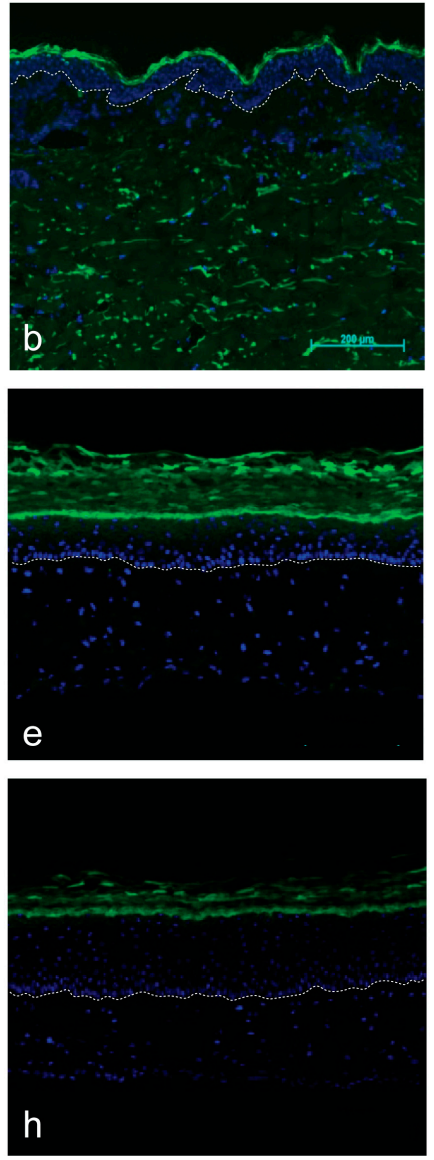

Keratin 14
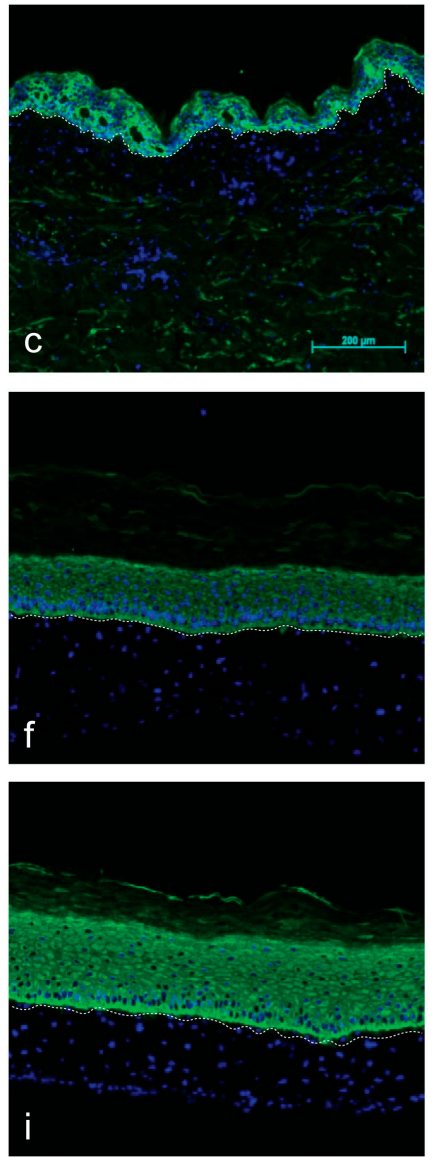

Figure 3. Immunofluorescence of differentiation markers of NHS (a-c), HS (d-f), and PS (g-i): Involucrin $(\mathbf{a}, \mathbf{d}, \mathbf{g})$, filaggrin $(\mathbf{b}, \mathbf{e}, \mathbf{h})$, and keratin $14(\mathbf{c}, \mathbf{f}, \mathbf{i})$. Healthy or psoriatic fibroblasts and keratinocytes were used to produce the skin substitutes $(\mathbf{d}-\mathbf{i})$. Dermo-epidermal junction is identified by the dotted line. The nuclei were stained with Hoechst. (Scale bar $=200 \mu \mathrm{m}$ ). 


\subsection{Percutaneous Absorption of Tazarotene}

An average of $8.46 \mathrm{mg}$ of Tazorac ${ }^{\circledR} 0.1 \%$ (8.46 $\mu \mathrm{g}$ of tazarotene) was deposited on the surface of each sample and the receptor medium was collected at $2 \mathrm{~h}, 4 \mathrm{~h}, 8 \mathrm{~h}$, and $24 \mathrm{~h}$. It appeared that more than $90 \%$ of the tazarotene was found in the wash samples of NHS and less than $1 \%$ was metabolized (Figure $4 \mathrm{a}, \mathrm{b}$ ). In HS and PS, tazarotene was found in washes (HS $=25-47 \%$ and PS = $22-47 \%$ ), epidermis ( $\mathrm{HS}=9-29 \%$ and PS $=6-27 \%$ ), and dermis (HS $=4-9 \%$ and PS $=1-11 \%$ ), showing a considerable amount of tazarotene absorbed by the skin (Figure $4 \mathrm{c}, \mathrm{d}$ ). The tazarotenic acid was found in epidermis ( $\mathrm{PS}=0.0015-0.0058 \mu \mathrm{g})$ and dermis $(\mathrm{PS}=0.0063-0.0476 \mu \mathrm{g})($ Figure $4 \mathrm{e}, \mathrm{f})$. Almost $1 \%$ of the dose was metabolized as tazarotenic acid in both conditions. Results of percutaneous absorption showed that no molecules reached the receptor compartment in these experimental conditions for all skin samples.
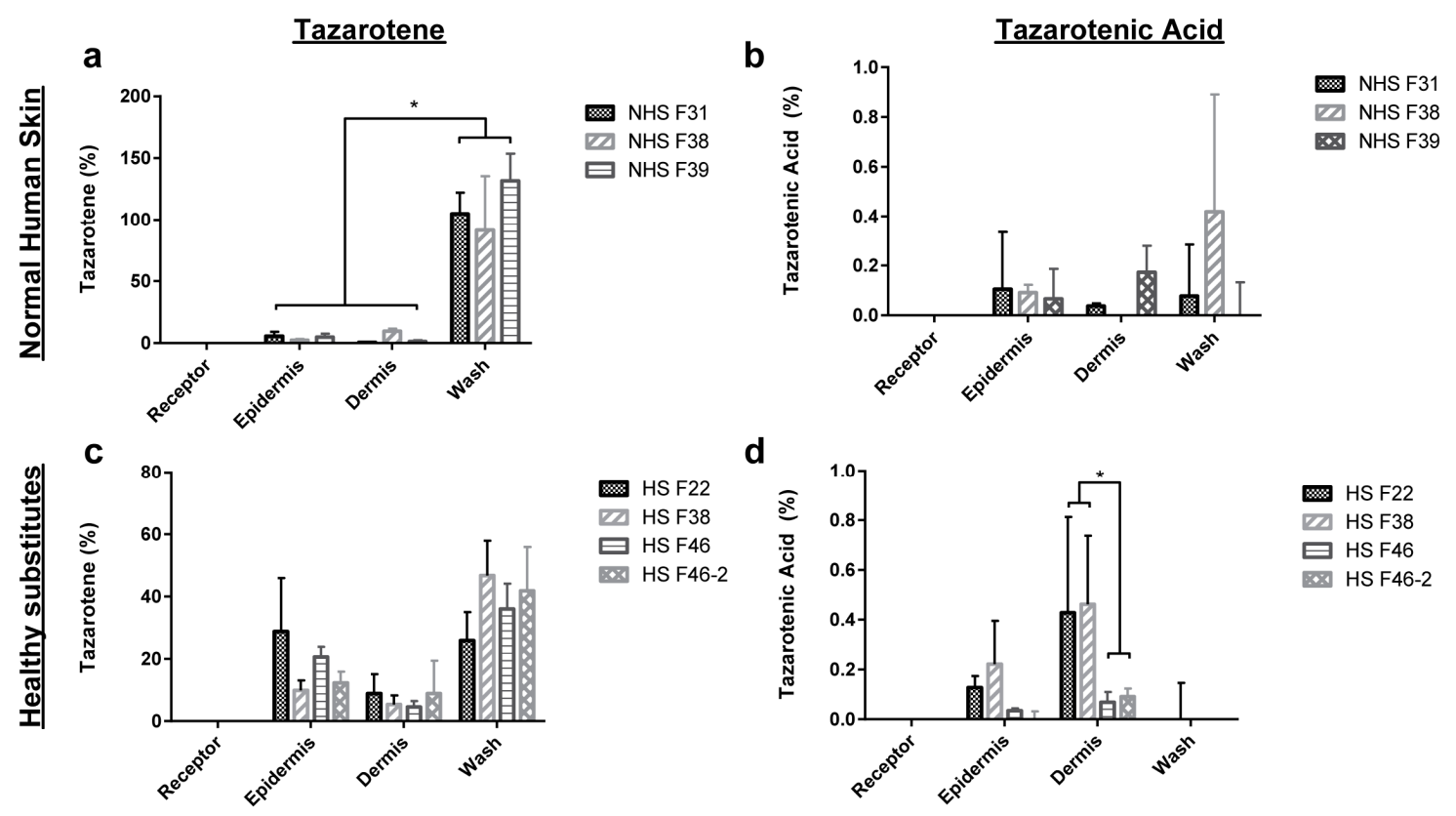

d
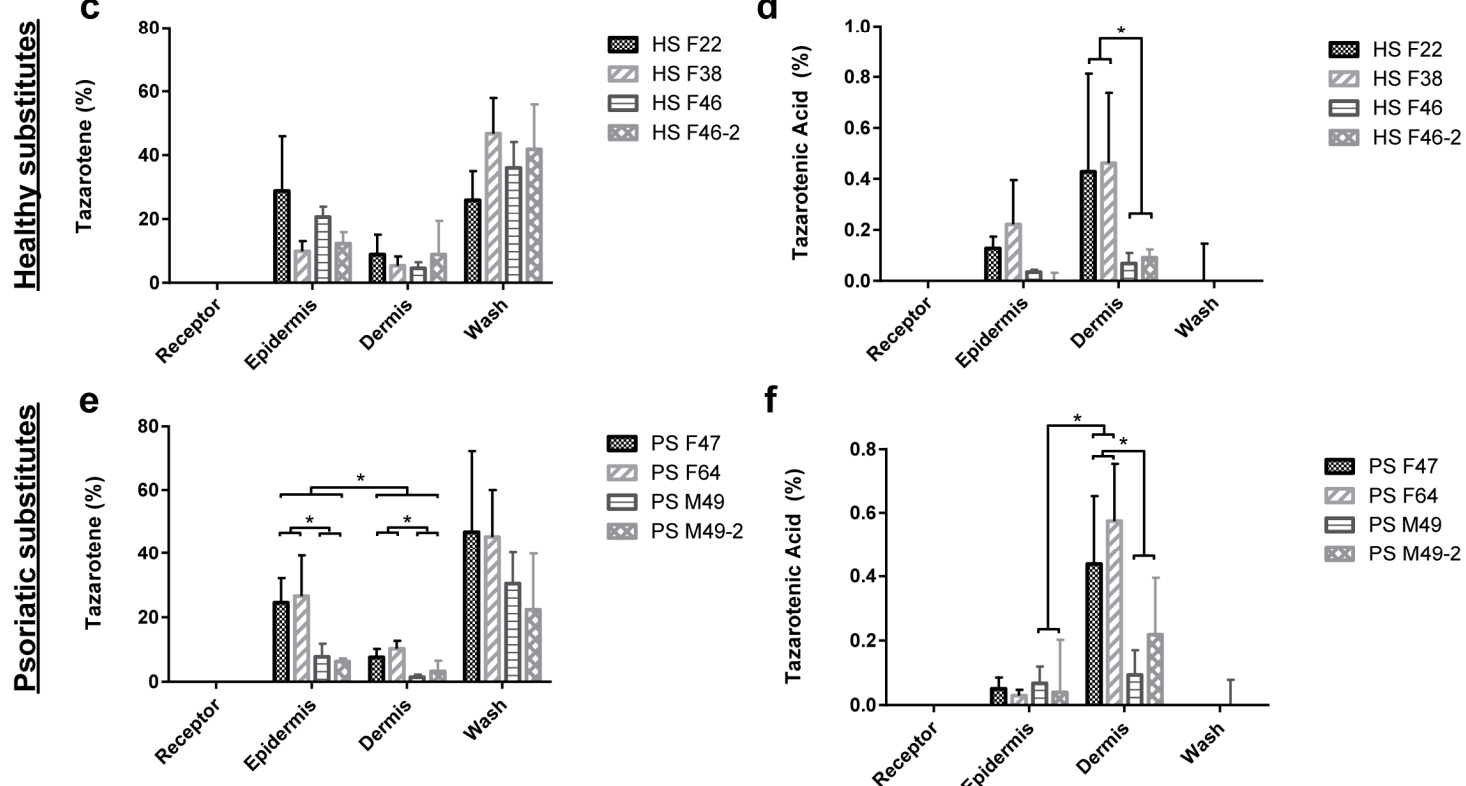

f

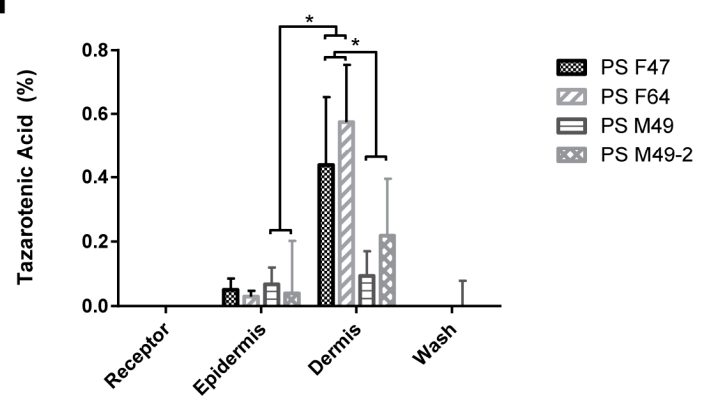

Figure 4. Amount (\%) of tazarotene and tazarotenic acid found in the receptor medium (DMEM without phenol red), epidermis, dermis, and wash after $24 \mathrm{~h}$ of percutaneous absorption. The metabolic capacity of normal human skin $(\mathbf{a}, \mathbf{b})$ was evaluated using skin samples of three donors while the metabolic capacity of healthy (HS, c,d) and psoriatic (PS, e,f) substitutes were evaluated on substitutes produced using cells of four donors. Donors are identified in legends by their sex (F: Female, M: Male) and age $\left(\mathrm{n}=\right.$ six per donors). Two-way ANOVA followed by Tukey's post-hoc test. ${ }^{*} p<0.05$.

\section{Discussion}

The need for innovative and effective tools to evaluate new dermopharmaceutical formulations is essential. The purpose of this project was to characterize the metabolic capacity of our unique in vitro screening platform in order to make it a suitable tool for dermopharmacological testing. Our expertise 
in skin tissue engineering allowed us to reconstruct a psoriatic skin model that can efficiently mimic the main aspects of the psoriasis phenotype. This study showed that this psoriatic skin model can also metabolize tazarotene. Our approach based on the development of a tissue-engineered skin made of patients' own cells obtained from a small skin biopsy of a psoriatic plaque could be a promising tool for more personalized medicine.

Macroscopic, histological, and immunofluorescence analyses (Figures 2 and 3) displayed a pathological skin phenotype. Histological analyses showed an increase in the living epidermis thickness in the psoriatic substitutes (Figure 2c-e), confirming acanthosis [27]. Moreover, early differentiation markers, such as involucrin and keratin 14, are overexpressed in pathologies presenting abnormal proliferation and differentiation processes, while late differentiation markers, such as filaggrin, are underexpressed [28,29]. Expression of these proteins is indeed deregulated compared to NHS and HS controls (Figure 3). Psoriatic skin substitutes reconstructed according to the self-assembly method presented these same features as seen in in vivo psoriasis, confirming their hyperproliferative and accelerated differentiation characteristics (Figure 3) [27,30]. Acanthosis, parakeratosis, and hyperproliferation are major psoriasis hallmarks. From these morphological features, a recent review by Niehues et al. listed the best-validated models as well as the expression of genes and key proteins [31]. Our psoriatic skin substitutes appear as the only one responding to these three morphological characteristics among the models listed. Moreover, many of the models listed use normal cells instead of pathological cells, highlighting further the effectiveness of our model. More recently, we validated our model by gene expression analysis of the skin substitutes, which revealed similar transcriptomic alterations to those found in psoriatic native skin [32].

The Franz diffusion cell system is recognized as an excellent tool for performing in vitro studies on transdermal drug diffusion [33-35]. The metabolic activity of the reconstructed skin substitutes, investigated by the detection of tazarotene biotransformation into tazarotenic acid, showed that tazarotene can diffuse through the stratum corneum. In fact, a significant amount of the molecule was found in the epidermis and the dermis of both healthy and psoriatic skin substitutes after $24 \mathrm{~h}$. The levels of tazarotene found in the skin substitutes seemed higher than levels in normal human skin. It is well known that NHS have a better skin barrier than skin substitutes [36]. Presence of tazarotenic acid in the epidermis and the dermis of healthy and psoriatic skin substitutes confirms the metabolic capacity of both skin models (Figure 4). To our knowledge, this study is the first to report such metabolic activity in tissue-engineered reconstructed skin models. These results are in accordance with results obtained in vivo where levels similar to $1 \%$ of the tazarotene dose was also metabolized into tazarotenic acid [37-39]. More tazarotene was found in the epidermis, while tazarotenic acid was found predominantly in the dermis. Localization in the skin of the esterase responsible for the tazarotene hydrolysis is controversial. Few studies suggest that esterases are only found in the living epidermis [40], while other studies also found them in the stratum corneum [41,42]. Most research on xenobiotic metabolization by esterases in human skin did not identify the esterase nature [43]. It is however known that serine-type esterases would be involved in blood and liver [44]. Therefore, conversion of hydrophobic prodrug tazarotene into its more hydrophilic metabolite could allow the tazarotenic acid to diffuse through the epidermis and then reach the dermis. In the present study, no tazarotene was detected in the receptor compartment of any skin samples after a 24-hour experiment under the experimental conditions (Figure 4).

Taken all together, these results have shown that skin substitutes reconstructed according to the self-assembly method could have the capacity to metabolize a psoriasis treatment and would be an efficient model to screen new antipsoriatic candidates. Studying the passage of substances through the skin using a non-invasive method on tissues allows a better understanding of the antipsoriatic activity of tested molecules. Furthermore, knowing the absorption rates and amounts of active ingredients and metabolites that diffuse through the skin can contribute to the increased knowledge of formulations in order to improve them. Moreover, this study showed promising avenues to overcome inter-individual variability in response to different treatments, which is one of the main challenges when finding 
a suitable treatment for patients. In the present study, levels of tazarotenic acid were significantly different between patients, showing different capacities to metabolize the drug in vitro. Therefore, our skin model could be used in clinic to screen in vitro responses of patient to a panel of drugs without having them experiencing the drawbacks of each drug.

In conclusion, this new in vitro model is a simple, rapid, easy-to-handle, and a cost-effective way to study in vitro absorption and metabolization of radiolabeled or non-radiolabeled molecules. These results indicate that our skin model can substitute for NHS in the selection of new drugs on the basis of their metabolism, efficacy, and/or safety.

Author Contributions: Conceptualization, A.M. and R.P.; methodology, A.M. and R.P.; investigation, A.M. and R.P.; data curation, A.M., M.S., G.R., A.G., S.M. and R.P.; writing—original draft preparation, A.M.; writing-review and editing, M.S., G.R., A.G., S.M and R.P.

Funding: This work was supported by the Canadian Institutes of Health Research (CIHR) (MOP-311262).

Acknowledgments: We thank Marielle Robert for her expertise and her help with the percutaneous absorption studies as well as Joël Provost and Laurence Giguère for their technical assistance with cell culture. A special thanks to Maxim Maheux for his help developing the UPLC method. We also thank Cindy Hayward for her help with the critical review of the manuscript. We acknowledge the Quebec Cell, Tissue and Gene Therapy Network-ThéCell (a thematic network supported by the ‘Fonds de recherche Québec-Santé' (FRQS)). Students received studentships from the FRQS (M Simard, A Grenier and S Morin), the 'Fonds d'Enseignement et de Recherche' (FER) of the Faculté de Pharmacie, Université Laval (M Simard, G Rioux, A Grenier and S Morin), the CHU de Québec-Université Laval (A Grenier and S Morin) and from the Centre de recherche en Organogénèse Expériementale de l'Université Laval/LOEX (G Rioux and A Grenier). R Pouliot is a career award scholar from the FRQS.

Conflicts of Interest: The authors declare no conflict of interest.

\section{References}

1. Raychaudhuri, S.; Farber, E. The prevalence of psoriasis in the world. J. Eur. Acad. Dermatol. Venereol. 2001, 15, 16-17. [CrossRef] [PubMed]

2. Freinkel, R.K.; Woodley, D.T. The Biology of the Skin; CRC Press: Boca Raton, FL, USA, 2001.

3. Guilhou, J.-J. Pathogénie du psoriasis. Med. Sci. 1993, 9, 412-416. [CrossRef]

4. Braun-Falco, O. The initial psoriatic lesion. In Proceedings of the Second International Symposium, Stanford, CA, USA, 12-15 July 1976; pp. 1-11.

5. Rongioletti, F.; Fiorucci, C.; Parodi, A. Psoriasis induced or aggravated by drugs. J. Rheumatol. 2009, 83, 59-61. [CrossRef] [PubMed]

6. Lowes, M.A.; Bowcock, A.M.; Krueger, J.G. Pathogenesis and therapy of psoriasis. Nature 2007, 445, 866-873. [CrossRef]

7. Lowes, M.A.; Suarez-Farinas, M.; Krueger, J.G. Immunology of psoriasis. Annu. Rev. Immunol. 2014, 32, 227-255. [CrossRef]

8. Duvic, M.; Nagpal, S.; Asano, A.T.; Chandraratna, R.A. Molecular mechanisms of tazarotene action in psoriasis. J. Am. Acad. Dermatol. 1997, 37, S18-S24. [CrossRef]

9. Wickett, R.R.; Visscher, M.O. Structure and function of the epidermal barrier. Am. J. Infect. Control 2006, 34, S98-S110. [CrossRef]

10. Nicolas, J.-F.; Thivolet, J. Psoriasis: De la Clinique à la Thérapeutique; John Libbey Eurotext: Montrouge, France, 1997; p. 241.

11. Lebwohl, M.G.; Bachelez, H.; Barker, J.; Girolomoni, G.; Kavanaugh, A.; Langley, R.G.; Paul, C.F.; Puig, L.; Reich, K.; van de Kerkhof, P.C. Patient perspectives in the management of psoriasis: Results from the population-based Multinational Assessment of Psoriasis and Psoriatic Arthritis Survey. J. Am. Acad. Dermatol. 2014, 70, 871-881. [CrossRef]

12. Chandra, A.; Ray, A.; Senapati, S.; Chatterjee, R. Genetic and epigenetic basis of psoriasis pathogenesis. Mol. Immunol. 2015, 64, 313-323. [CrossRef]

13. Gollnick, H.; Menter, A. Combination therapy with tazarotene plus a topical corticosteroid for the treatment of plaque psoriasis. Br. J. Dermatol. Suppl. 1999, 140, 18-23. [CrossRef]

14. Tanghetti, E.; Lebwohl, M.; Stein Gold, L. Tazarotene Revisited: Safety and Efficacy in Plaque Psoriasis and Its Emerging Role in Treatment Strategy. J. Drugs Dermatol. 2018, 17, 1280-1287. [PubMed] 
15. Barker, C.L.; Clothier, R.H. Human keratinocyte cultures as models of cutaneous esterase activity. Toxicol. In Vitro Int. J. Publ. Assoc. Bibra 1997, 11, 637-640. [CrossRef]

16. Kamili, Q.U.; Menter, A. Topical treatment of psoriasis. Curr. Probl. Dermatol. 2009, 38, 37-58. [CrossRef] [PubMed]

17. Talpur, R.; Cox, K.; Duvic, M. Efficacy and safety of topical tazarotene: A review. Expert Opin. Drug Metab. Toxicol. 2009, 5, 195-210. [CrossRef] [PubMed]

18. Nagpal, S.; Patel, S.; Asano, A.T.; Johnson, A.T.; Duvic, M.; Chandraratna, R.A.S. Tazarotene-Induced Gene 1 (TIG1), a Novel Retinoic Acid Receptor-Responsive Gene in Skin. J. Investig. Derm. 1996, 106, 269-274. [CrossRef] [PubMed]

19. Chandraratna, R.A.S. Tazarotene: The first receptor-selective topical retinoid for the treatment of psoriasis. J. Am. Acad. Dermatol. 1997, 37, S12-S17. [CrossRef]

20. Germain, L.; Rouabhia, M.; Guignard, R.; Carrier, L.; Bouvard, V.; Auger, F. Improvement of human keratinocyte isolation and culture using thermolysin. Burns 1993, 19, 99-104. [CrossRef]

21. Auger, F.A.; Valle, C.A.L.; Guignard, R.; Tremblay, N.; Noel, B.; Goulet, F.; Germain, L. Skin equivalent produced with human collagen. In Vitro Cell. Dev. Biol. Anim. 1995, 31, 432-439. [CrossRef]

22. Franz, T.J. Percutaneous absorption on the relevance of in vitro data. J. Investig. Dermatol. 1975, 64, $190-195$. [CrossRef]

23. Simard, M.; Julien, P.; Fradette, J.; Pouliot, R. Modulation of the Lipid Profile of Reconstructed Skin Substitutes after Essential Fatty Acid Supplementation Affects Testosterone Permeability. Cells 2019, 8. [CrossRef]

24. Iizuka, H.; Ishida-Yamamoto, A.; Honda, H. Epidermal remodelling in psoriasis. Br. J. Dermatol. 1996, 135, 433-438. [CrossRef] [PubMed]

25. Mils, V.; Basset-Séguin, N.; Molès, J.-P.; Tesnière, A.; Leigh, I.; Guilhou, J.-J. Comparative analysis of normal and psoriatic skin both in vivo and in vitro. Differentiation 1994, 58, 77-86. [CrossRef] [PubMed]

26. Stoler, A.; Kopan, R.; Duvic, M.; Fuchs, E. Use of monospecific antisera and cRNA probes to localize the major changes in keratin expression during normal and abnormal epidermal differentiation. J. Cell Biol. 1988, 107, 427-446. [CrossRef] [PubMed]

27. Van de Kerkhof, P.C. Textbook of Psoriasis, 2nd ed.; Wiley: Hoboken, NJ, USA, 2003.

28. Thewes, M.; Stadler, R.; Korge, B.; Mischke, D. Normal psoriatic epidermis expression of hyperproliferationassociated keratins. Arch Derm. Res 1991, 283, 465-471. [CrossRef] [PubMed]

29. Ramesh, R.; Jagannatha, R.K.; Basavaraj, K.H. A comprehensive review on biomarkers in psoriasis. Clin. Exp. Dermatol. 2009, 34, 658-663.

30. McKay, I.A.; Leigh, I.M. Altered keratinocyte growth and differentiation in psoriasis. Clin. Dermatol. 1995, 13, 105-114. [CrossRef]

31. Niehues, H.; van den Bogaard, E.H. Past, present and future of in vitro 3D reconstructed inflammatory skin models to study psoriasis. Exp. Dermatol. 2018, 27, 512-519. [CrossRef]

32. Rioux, G.; Pouliot-Berube, C.; Simard, M.; Benhassine, M.; Soucy, J.; Guerin, S.L.; Pouliot, R. The TissueEngineered Human Psoriatic Skin Substitute: A Valuable In Vitro Model to Identify Genes with Altered Expression in Lesional Psoriasis. Int. J. Mol. Sci. 2018, 19. [CrossRef]

33. Khan, G.M.; Frum, Y.; Sarheed, O.; Eccleston, G.M.; Meidan, V.M. Assessment of drug permeability distributions in two different model skins. Int. J. Pharm. 2005, 303, 81-87. [CrossRef]

34. Lombardi Borgia, S.; Schlupp, P.; Mehnert, W.; Schafer-Korting, M. In vitro skin absorption and drug release-A comparison of six commercial prednicarbate preparations for topical use. Eur. J. Pharm. Biopharm. 2008, 68, 380-389. [CrossRef]

35. Alonso, C.; Carrer, V.; Espinosa, S.; Zanuy, M.; Cordoba, M.; Vidal, B.; Dominguez, M.; Godessart, N.; Coderch, L.; Pont, M. Prediction of the skin permeability of topical drugs using in silico and in vitro models. Eur. J. Pharm. Sci. Off. J. Eur. Fed. Pharm. Sci. 2019, 136, 104945. [CrossRef] [PubMed]

36. Supp, D.M.; Boyce, S.T. Engineered skin substitutes: Practices and potentials. Clin. Dermatol. 2005, 23, 403-412. [CrossRef] [PubMed]

37. Su, Y.-H.; Fang, J.-Y. Drug delivery and formulations for the topical treatment of psoriasis. Expert Opin. Drug Deliv. 2008, 5, 235-249. [CrossRef] [PubMed]

38. Diane, D.-S.; Matsumoto, R.M.; Usansky, J.I. Clinical pharmacokinetics and drug metabolism of tazarotene. Clin. Pharmacokinet. 1999, 37, 273-287. 
39. Li, L.; Zhang, M.; Ma, P.; Qian, K. Development of a simple method for simultaneous determination of tazarotene and betamethasone dipropionate and their metabolites using LC-MS method and its application to dermatopharmacokinetic study. Biomed. Chromatogr. 2019, 33, e4557. [CrossRef] [PubMed]

40. Hewitt, P.G.; Perkins, J.; Hotchkiss, S.A.M. Metabolism of Fluroxypyr, Fluroxypyr Methyl Ester, and the Herbicide Fluroxypyr Methylheptyl Ester. I: During Percutaneous Absorption through Fresh Rat and Human Skin In Vitro. Drug Metab. Dispos. 2000, 28, 748-754.

41. Sondell, B.; Thornell, L.-E.; Egelrud, T. Evidence That Stratum Corneum Chymotryptic Enzyme Is Transported to the Stratum Corneum Extracellular Space Via Lamellar Bodies. J. Investig. Dermatol. 1995, 104, 819-823. [CrossRef]

42. Beisson, F.; Aoubala, M.; Marull, S.; Moustacas-Gardies, A.-M.; Voultoury, R.; Verger, R.; Arondel, V. Use of the Tape Stripping Technique for Directly Quantifying Esterase Activities in Human Stratum Corneum. Anal. Biochem. 2001, 290, 179-185. [CrossRef]

43. Oesch, F.; Fabian, E.; Guth, K.; Landsiedel, R. Xenobiotic-metabolizing enzymes in the skin of rat, mouse, pig, guinea pig, man, and in human skin models. Arch. Toxicol. 2014, 88, 2135-2190. [CrossRef]

44. Madhu, C.; Duff, S.; Baumgarten, V.; Rix, P.; Small, D.; Tang-Liu, D. Metabolic deesterification of tazarotene in human blood and rat and human liver microsomes. J. Pharm. Sci. 1997, 86, 972-974. [CrossRef]

(C) 2019 by the authors. Licensee MDPI, Basel, Switzerland. This article is an open access article distributed under the terms and conditions of the Creative Commons Attribution (CC BY) license (http://creativecommons.org/licenses/by/4.0/). 